\title{
Treatment of digit ulcers in a patient with Buerger's disease by using cervical spinal cord stimulation -a case report-
}

\author{
Sang-Wook Ryu, Hee-Jung Jeon, Sam-Soon Cho, Rak-Min Choi, Jin-Sun Yoon, Hong-Seok Ko, and \\ Jae-Do Lee
}

Department of Anesthesiology and Pain Medicine, Veterans Health Service Medical Center, Seoul, Korea

Buerger's disease (thromboangiitis obliterans) is known as a segmental inflammatory vasculitis that involves the smallsized and medium-sized arteries, veins, and nerves. Most effective treatment for Buerger's disease is smoking cessation. Except for the cessation of tobacco use, surgical revascularization is available in severe ischemia and a distal target vessel. Amputation has been used as the last treatment option of the disease up to the present. Increasing limb survival and decreasing amputation rate is important. This case describes the use of spinal cord stimulation (SCS) in patient with Buerger's disease and its effect is not only the complete healing of ulcers but also amputation is not performed. (Korean J Anesthesiol 2013; 65: 167-171)

Key Words: Thromboangiitis obliterans, Electric stimulation, Ulcer.

Buerger's disease is associated with extreme pain, and despite various treatment methods, about $20 \%$ of patients undergo amputation of limbs, which leads to low quality of life and significantly affects socioeconomic position. Complete smoking cessation is the primary prevention and treatment method to stop disease progression in Buerger's disease patients, while active treatments such as combination drug therapy, arterial bypass surgery, and sympathetic block are also considered simultaneously. Combination drug therapy is known to be effective for pain relief but does not make a significant difference for ischemic ulcers, and vasculitis can continue to progress even after arterial bypass surgery. Sympathetic block can be chosen to treat ischemic ulcers but does not cause a decrease in the limb amputation rate [1].

A patient with Buerger's disease presented with severe ulcers that showed no response to medications and sympathetic block. A limb amputation was planned, but the patient refused the amputation and was referred to a pain clinic. Cervical spinal cord stimulation was conducted, and the patient experienced effective pain relief and complete recovery of the ulcers. The author reports this case along with a literature review.

Received: June 19, 2012. Revised: 1st, August 23, 2012; 2nd, September 11, 2012; 3rd, September 14, 2012. Accepted: September 14, 2012.

Corresponding author: Jae-Do Lee, M.D., Department of Anesthesiology and Pain Medicine, Veterans Health Service Medical Center, 53, Jinhwang-doro 61gil, Gangdong-gu, Seoul 134-791, Korea. Tel: 82-2-2225-4060, Fax: 82-2-2225-1704, E-mail: devlin@naver.com 두 This is an open-access article distributed under the terms of the Creative Commons Attribution Non-Commercial License (http:// creativecommons.org/licenses/by-nc/3.0/), which permits unrestricted non-commercial use, distribution, and reproduction in any medium, provided the original work is properly cited. 


\section{Case Report}

The 62-year-old male patient in this case report had a smoking history of more than 30 pack-years and a medical history of hypertension and diabetes. He had had a percutaneous coronary intervention 6 years previously due to angina and had noticed a cold and numbing sensation in the extremities of both hands for the previous 3 years. Two years previously, the patient's fourth finger on the left hand had experienced blue discoloration, with extreme pain of greater than $90 / 100 \mathrm{~mm}$ on the visual analogue scale (VAS), followed by ulceration. After a few tests at the orthopedic clinic, the patient had been diagnosed with Buerger's disease. He had been referred to the pain clinic for conservative treatment one year previously. On the patient's upper extremity angiography, greater than $80 \%$ stenosis was observed in the first and third finger artery in the area of radial artery and palmer arch, and greater than $90 \%$ stenosis was also observed in distal ulnar artery.

At the time of referral, the patient had been taking oxycodone $40 \mathrm{mg}$ twice a day, as well as limaprost $5 \mu \mathrm{g}$ and gabapentin $300 \mathrm{mg} 3$ times day. Even with an increase in opiate dose and several chest sympathetic block and stellate ganglion blocks, the analgesic effect was temporal. The pain and ulcers on the fingers worsened, and a finger amputation was planned as arterial bypass surgery was not a valid method for this patient. Although a number of treatments were tried, the patient complained about extreme pain, $90 / 100 \mathrm{~mm}$ on VAS, and there were severe gangrenous ulcers progressing on the fourth finger of the left hand and the index finger of the right hand. The patient was very much against the amputation even though he was under extreme pain and had been referred to the pain clinic several times. Hence, the decision was made to attempt spinal cord stimulation prior to amputation.

The blood pressure, heart rate, oxygen saturation, and electrocardiography were monitored, and, with the patient in the prone position, local anesthesia was performed at T2-3 intervertebral space. A 15-gauge Tuohy needle was used for the paramedian approach with a $\mathrm{C}$-arm fluoroscopic image. After ensuring the needle was positioned in the epidural space, the guidewire was inserted for easy insertion of the electrode, and the electrode was positioned in the posterior epidural space using the $\mathrm{C}$-arm fluoroscopic image. The guidewire was removed, and the octrode electrode lead (Advanced Neuromodulation System Inc, Plano, Texas, USA) was placed $2 \mathrm{~mm}$ left of the radiological center. The distal electrode was placed at the bottom of the cervical spine (Fig. 1).

The electrode was connected to the test stimulator, and the stimulation was profound in the areas with pain. After the test stimulation, the pain was reduced by $50 \%$. During 1 week of test stimulation, the patient's pain was maintained to within 20-30/100 mm on VAS, and the use of opiate analgesics was decreased by $50 \%$. Hence, the permanent spinal cord stimulation insertion procedure was carried out. The Genesis IPG (Advanced Neuromodulation System Inc, Plano, Texas, USA) was buried under the lower left-side clavicle, and stimulation was well controlled with 3-4+ electrode combination, $4.0 \mathrm{~V}$ amplitude, $240 \mathrm{msec}$ pulse width, and $34 \mathrm{~Hz}$ frequency.

After the spinal cord stimulation insertion, the ulcers on both hands began gradual recovery and were completely recovered after 6 months. The pain was maintained within 30/100 mm on VAS, and the use of opioid analgesics was decreased more than $50 \%$ compared with the pre-procedure stage (Fig. 2).
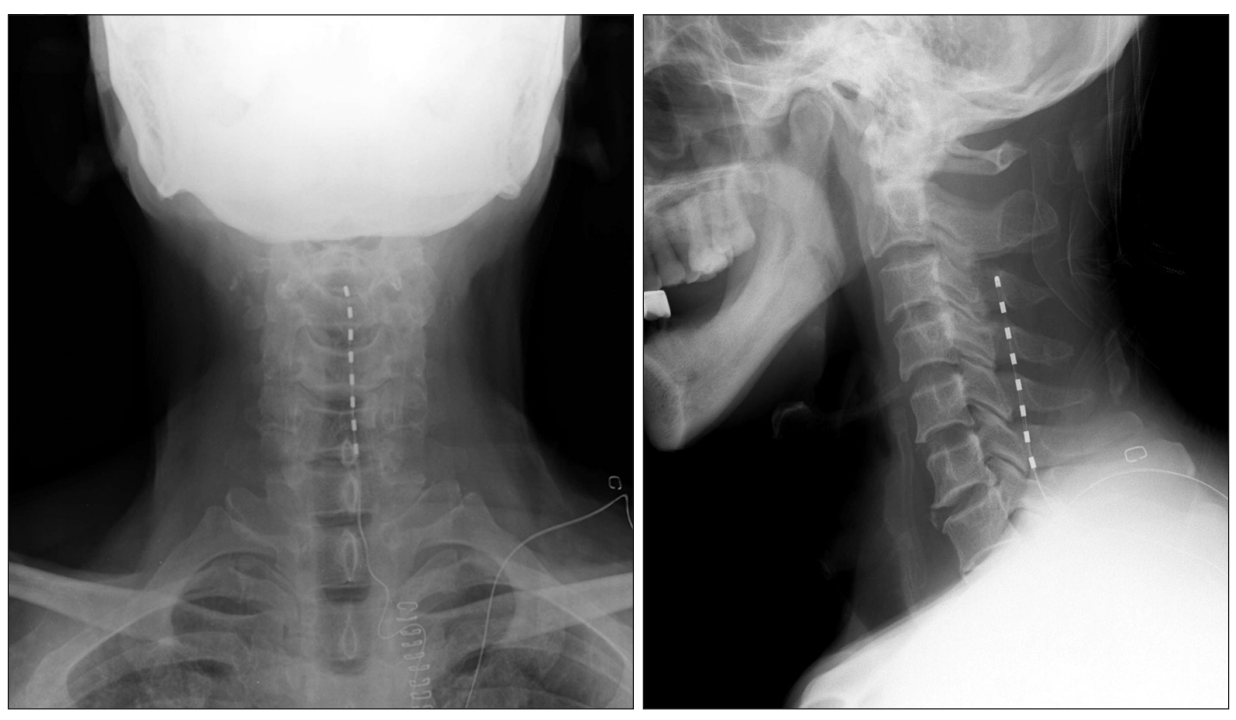

Fig. 1. The tip of electrode located at the 3 rd cervical vertebral level. 

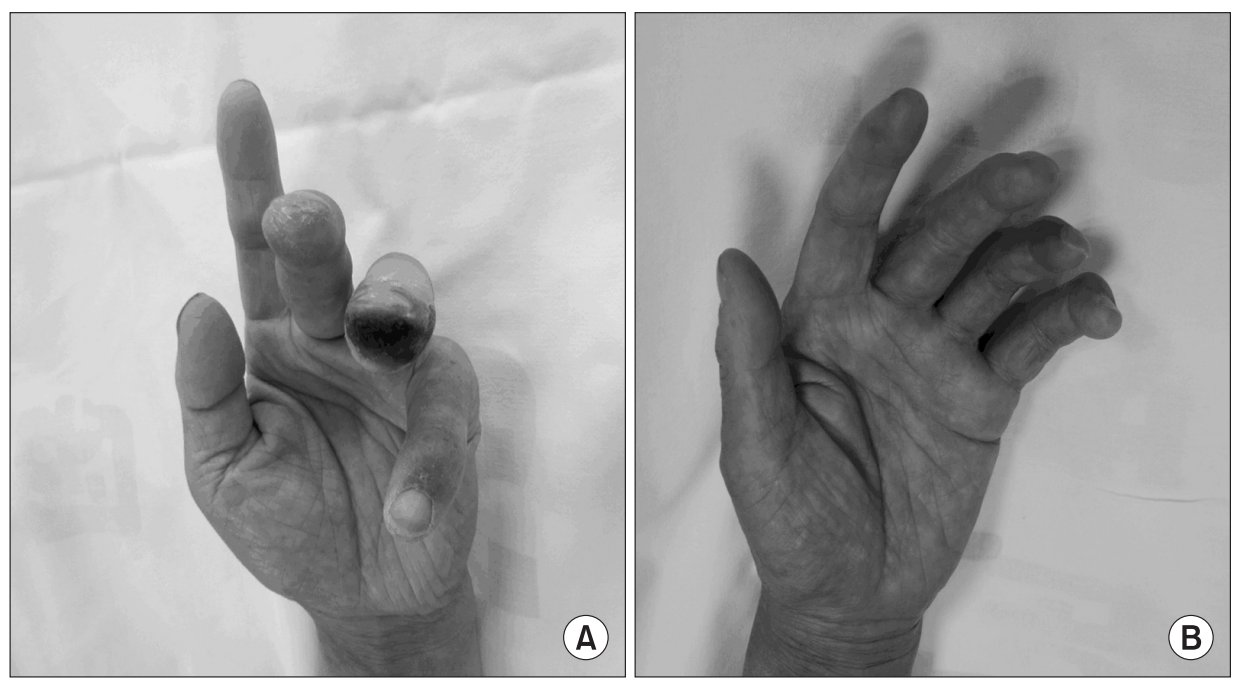

Fig. 2. (A) Patient has shallow ulceration on the third finger and deep ishchemic ulceration on the fourth finger of the left hand. (B) The ischemic ulcers completely healed 6 months after implantation of a permanent spinal cord stimulator.

\section{Discussion}

The cause of Buerger's disease is unknown, but smoking is considered to be a major factor in the initiation and progression of the disease. The progression of the disease is relatively fast, with 2-5 years typically passing between the onset of the disease and loss of tissues. Therefore, the foundation of treatment is smoking cessation to stop the disease progression and prevent amputation of the lesions. Other than smoking cessation, systemic treatments such as thrombolytic agents, anticoagulants and prostaglandin agents can be used, as well as direct arterial bypass surgery, sympathectic block, and amputation [1-4].

A prostaglandin agent has been used in occlusive arterial disease due to its vasodilation and antiplatelet effects, but it has the disadvantage of $80-90 \%$ loss of efficacy through lung circulation when it is administered intravenously [2]. Bypass surgery or percutaneous angioplasty tend to limit the treatment, as the saphenous vein for the graft can also accompany phlebitis, which often continues to progress even after the bypass surgery, and the lesions generally spread widely [4]. Sympathectic nerve block is known to be effective for the ischemic ulcer but does not decrease the rate of the most severe limb amputations [3]. According to a number of studies, spinal cord stimulation in patients with Buerger's disease is not only a safe treatment method but also can prevent limb amputation, reduce pain, and improve blood flow and ulceration $[5,6]$.

Spinal cord stimulation in Buerger's disease reduces ischemic pain and the rate of amputation by treating the ulceration. Hence, it is recommended for people who fail conservative or surgical treatment and for whom arterial bypass surgery is not feasible, as well as for people who complain about severe ischemic pain after failure of medication [7,8]. Donas et al. [1] performed spinal cord stimulation in 29 patients with Buerger's disease who did not respond to medications or arterial bypass surgery and followed up for up to 4 years. Most of the patients (limb salvage rate $93.1 \%$ ) presented with a significant increase in microcirculation of the blood vessels. Lower limb amputation, one of the most serious problems in Buerger's disease treatment, was performed in only 2 patients, a considerably lower rate than that seen in other studies that conducted sympathectic block or aterial bypass surgery. A number of other studies have suggested spinal cord stimulation in Buerger's disease as a safe treatment method that can reduce the pain and improve blood flow and the treatment of ulcers. Therefore, it has been argued that spinal cord stimulation should be considered as an important alternative treatment choice $[5,6]$.

The reduction in the rate of limb amputation due to spinal cord stimulation is hugely beneficial in socioeconomic terms. The cross-sectional problem of the increase in treatment costs due to spinal cord stimulation can easily be offset by decreases in the length of hospital stay after the procedure, prevention of amputation, and the increase in quality of life due to pain reduction. Therefore, spinal cord stimulation should be considered in patients who do not find a sympathectic block to be effective in ischemic pain relief or for whom bypass surgery is not indicated. However, spinal cord stimulation is a high-cost treatment and only should be considered in patients with a high severity of disease, consistent symptoms, lack of success with medication, and no indication for arterial bypass surgery [9].

In 1976, Cook et al. [7] reported using electronic stimulation of spinal nerves and the dorsal root in vascular disease of limbs, and since then spinal cord stimulation has been successfully carried out in the treatment of atherosclerotic and vasospastic peripheral arterial disease and is mainly used in vascular diseases of lower limbs. There is no reported case of spinal cord stimulation in a patient with Buerger's disease in Korea. A case 
has been reported in which spinal cord stimulation was carried out in a 60-year-old male patient with Raynaud's syndrome, and improvements in pain and ulceration were seen although the ulceration was not completely cured [10].

Spinal cord stimulation improves microcirculation by inhibiting sympathetic vasoconstriction and stimulates the secretion of a number of inhibitory neurotransmitters, leading to a reduction in pain in patients with Buerger's disease. Spinal cord stimulation reduces pain in Buerger's disease by promoting the secretion of gamma-aminobutyric acid (GABA), serotonin, and substance $\mathrm{P}$ at the spinal nerve dorsal horn. In addition, it inhibits nociceptive neurotransmission, and intramedullary oxidative nitric oxide and GABA act as an important mediator for the induction of pain relief after spinal cord stimulation [11-13]. Spinal cord stimulation in peripheral obstructive arterial disease can treat chronic ischemic pain and ulcers and also prevent amputation. Unlike the pain reduction, the mechanism of action for the improvement in microcirculation is unknown, and the improvement in ulceration is considered to be the outcome of the reduction in vasoconstriction due to the inhibition of peripheral sympathetic nerves. The improvement in microcirculation after spinal cord stimulation in patients with peripheral obstructive arterial disease can be quantified by the ankle-brachial index, transcutaneous oxygen pressure, and regional perfusion index. It is simple and non-invasive and can effectively measure the degree of blood circulation of cutaneous tissue [14,15].

In this case, the progression of ulcers was only observed through gross examination before and after the procedure, and objective measures were not carried out to determine the degree of microcirculation. Also, angiography was not carried out after spinal cord stimulation, and thus the change in peripheral vessels could not be confirmed. However, there was a reduction in redness on the patient's palms immediately after the spinal cord stimulation, and significant improvement was seen a week after the procedure. The ulceration on both hands seemed to improve slightly one week after the procedure, and the ulceration gradually improved to achieve complete recovery 6 months after the procedure.

Although the patient fully recovered from the ulceration within 6 months of spinal cord stimulation, the pain still was present. However, the pain decreased by about $50 \%$ from $90 / 100$ $\mathrm{mm}$ on VAS immediately after the procedure and substantially improved after 6 months to $20-30 / 100 \mathrm{~mm}$ on VAS. This pain reduction led to more than a $50 \%$ reduction in the use of opiate analgesics and the total amount of medication. After spinal cord stimulation, the patient completely recovered from ulcers on fingers of both hands and achieved a significant reduction in pain, although the cold sensation in his hands remained. Ultimately, the patient was able to return to work without any functional impairment.

Therefore, the authors suggest that spinal cord stimulation be considered in patients with Buerger's disease who do not response to medications or sympathectic block and for whom arterial bypass surgery is not feasible. Spinal cord stimulation would be an excellent treatment choice in patients with Buerger's disease when an amputation is planned due to the lack of successful response to various treatments.

\section{References}

1. Donas KP, Schulte S, Ktenidis K, Horsch S. The role of epidural spinal cord stimulation in the treatment of Buerger's disease. J Vasc Surg 2005; 41: 830-6.

2. Oral iloprost in the treatment of thromboangiitis obliterans (Buerger's disease): a double-blind, randomised, placebo-controlled trial. The European TAO Study Group. Eur J Vasc Endovasc Surg 1998; 15: 300-7.

3. De Giacomo T, Rendina EA, Venuta F, Lauri D, Mercadante ES, Anile M, et al. Thoracoscopic sympathectomy for symptomatic arterial obstruction of the upper extremities. Ann Thorac Surg 2002; 74: 885-8.

4. Shionoya S. Buerger's disease: diagnosis and management. Cardiovasc Surg 1993; 1: 207-14.

5. Horsch S, Schulte S, Hess S. Spinal cord stimulation in the treatment of peripheral vascular disease: results of a single-center study of 258 patients. Angiology 2004; 55: 111-8.

6. Kay AD, McIntyre MD, Macrae WA, Varma TR. Spinal cord stimulation--a long-term evaluation in patients with chronic pain. Br J Neurosurg 2001; 15: 335-41.

7. Cook AW, Oygar A, Baggenstos P, Pacheco S, Kleriga E. Vascular disease of extremities. Electric stimulation of spinal cord and posterior roots. N Y State J Med 1976; 76: 366-8.

8. Augustinsson LE, Linderoth B, Mannheimer C, Eliasson T. Spinal cord stimulation in cardiovascular disease. Neurosurg Clin N Am 1995; 6: 157-65.

9. Swigris JJ, Olin JW, Mekhail NA. Implantable spinal cord stimulator to treat the ischemic manifestations of thromboangiitis obliterans (Buerger's disease). J Vasc Surg 1999; 29: 928-35.

10. Kim SJ, Kang G, Lee MG, Lee MK, Choi SS. Effective treatment of a digital ulcer and pain in a Raynaud's syndrome patient with using a 
spinal cord stimulator : A case report. Anesth Pain Med 2009; 4: 11-4.

11. Croom JE, Foreman RD, Chandler MJ, Koss MC, Barron KW. Role of nitric oxide in cutaneous blood flow increases in the rat hindpaw during dorsal column stimulation. Neurosurgery 1997; 40: 565-70.

12. Linderoth B, Stiller CO, Gunasekera L, O'Connor WT, Franck J, Gazelius B, et al. Release of neurotransmitters in the CNS by spinal cord stimulation: survey of present state of knowledge and recent experimental studies. Stereotact Funct Neurosurg 1993; 61: 157-70.

13. Stanton-Hicks M, Salamon J. Stimulation of the central and peripheral nervous system for the control of pain. J Clin Neurophysiol 1997; 14: 46-62.

14. Hauser CJ, Shoemaker WC. Use of a transcutaneous PO2 regional perfusion index to quantify tissue perfusion in peripheral vascular disease. Ann Surg 1983; 197: 337-43.

15. Scheffler A, Rieger H. Clinical information content of transcutaneous oxymetry (tcpO2) in peripheral arterial occlusive disease (a review of the methodological and clinical literature with a special reference to critical limb ischaemia). Vasa 1992; 21: 111-26. 